\title{
Correlation Studies between Weather Parameters and Sesamum (Sesamum indicm L.) Varieties Yield at Different Phenophases under Change in Climate
}

\author{
D.M. Sawant ${ }^{1 *}$, U.N. Alse ${ }^{2}$, B.V. Asewar ${ }^{3}$ and A.M. Khobragade ${ }^{4}$ \\ ${ }^{1}$ Department of Agriculture Meteorology, Parbhani, India \\ ${ }^{2}$ ATIC, VNMKV, Parbhai, India \\ ${ }^{3}$ Dry land Agriculture Research Center, VNMKV, Parbhai, India \\ ${ }^{4}$ Agriculture Meteorology, VNMKV, Parbhani, India \\ *Corresponding author
}

\section{A B S T R A C T}

\section{Keywords \\ Sesamum, Yield, Correlation, Climate Change \\ Article Info \\ Accepted: \\ 10 April 2019 \\ Available Online: \\ 10 May 2019}

An experiment was carried out at Agricultural Meteorology Department farm, College of Agriculture, Vasantrao NaikMarathwada Krishi Vidyapeeth, Parbhani, during kharif season 2016. The field experiment was laid out in the split spot design with three replication and treatment combination of four sowing dates viz., D1 (27 MW), D2 (28 MW), D3 (29 MW) and D4 (30 MW) as main plot and four genotypes Phule til -1, Gujarat - 2, JLT - 408 and JLT -7 as sub-plot treatment. The crop was sown with spacing $45 \times 15$ $\mathrm{cm}$ on $5.4 \times 4.0 \mathrm{~m}^{2}$ gross plot size and $4.5 \times 3.6 \mathrm{~m}^{2}$ net plot size. Among the Sowing dates and varieties, the variety JLT -408 when sown during $28^{\text {th }} \mathrm{MW}$ was significantly superior over the other varieties and sowing dates. The result of the study indicated that correlation of rainfall, rainy day, RH-II at P1 (Sowing dates to vegetative), P2 (vegetative to reproductive) and rainfall, rainy day, $\mathrm{RH}-\mathrm{I}, \mathrm{RH}-\mathrm{II}$ at P3 (reproductive to maturity), P4 (maturity to harvesting) stage was positively correlated with sesamum yield. Tmaxi., Tmini was negatively correlated with sesamum yield at all phases expected Tmini at P3 stage in variety JLT -408.

\section{Introduction}

Sesamum [Sesamum indicum L.] belongs to the family Pedaliaceae and is one of the most ancient oilseed crop and used in cooking. Crop also cultivated either as a pure stand or as a mixed crop with aus rice, jute, groundnut, millets and sugarcane. Sesamum is basically considered a crop of warm region of tropics and subtropics. It requires fairly hot condition during growth to produce maximum yield. A temperature of $25-27^{0} \mathrm{C}$ encourage rapid germination, initial growth and flower formation. In India, it is cultivated on an area about 1746.06 thousand hectares with production 827.83 thousand meteric tones and productivity $474 \mathrm{~kg} \mathrm{ha}^{-1}$ (Anonymous, 2015).

In Maharashtra it is cultivated on an area about 204 hundred hectares with production 42 hundred tones and productivity $505 \mathrm{~kg} \mathrm{ha}^{-1}$ (Anonymous, 2015). The distribution of 
rainfall in monsoon decides the yield of rainfed crops.

\section{Materials and Methods}

A field experiment was conducted an experiment was conducted in split plot design with three replications. Treatment under study were four sowing dates in kharif season i.e. D1 (27th MW), D2 (28th MW), D3 (29th MW) and D4 (30th MW), and four different varieties Phule til -1,Gujarat - 2, JLT - 408 and JLT - 7 were sown with spacing $45 \times 15$ $\mathrm{cm}$.

The gross plot size was $5.4 \times 4.0 \mathrm{~m}^{2}$ and net plot size was $4.5 \times 3.6 \mathrm{~m}^{2}$ during the kharif season of 2016-17 under rainfed condition on the experimental farm of the Department of Agricultural Meteorology, College of Agriculture, Vasantrao Naik Marathwada Krishi Vidyapeeth, Parbhani. The sowing of seed was done by dibbling method on respective date of sowing. Observations were recorded on five plants randomly selected per treatment.

\section{Correlation studies}

Simple correlation between weather parameters i.e. Rainfall, Rainy days, Maximum temperature, Minimum temperature, relative humidity, Evaporation, Bright sun shine hours and wind velocity on the development of sesamum was estimated to know the correlation between these weather parameters and sesamum yield.

The procedure and formula described by Snedecor and Cochran (1968) were adopted and significance was tested.
Where,

$\mathrm{r}=$ Correlation coefficient

$\mathrm{x}=$ Independent variable (attributes)

$\mathrm{y}=$ Dependent variable (yield).

\section{Results and Discussion}

\section{Weather conditions during the crop growing season}

The total rainfall recorded during growing period $\left(27^{\text {th }} \mathrm{MW}\right.$ to $\left.44^{\text {th }} \mathrm{MW}\right)$ of sesamum crop was $990.7 \mathrm{~mm}$ in 45 rainy days. During the crop growing period the highest maximum temperature $\left(32.5^{\circ} \mathrm{C}\right)$ recorded in $29-\mathrm{MW}$ and lowest maximum temperature $\left(28.2^{\circ} \mathrm{C}\right)$ recorded in 31-MW respectively. Whereas the highest minimum temperature $\left(23.9^{\circ} \mathrm{C}\right)$ recorded in $27-\mathrm{MW}$ and lowest minimum temperature $\left(14.4^{\circ} \mathrm{C}\right)$ recorded in $44-\mathrm{MW}$, respectively.

The morning time relative humidity ranged between 74 to 96 per cent while evening relative humidity ranged between 31 to 85 per cent during crop growth period. The highest evaporation during the crop growing season $(5.9 \mathrm{~mm})$ in $43-\mathrm{MW}$ and lowest $(2.5 \mathrm{~mm})$ was recorded in 31-MW respectively. The crop season experienced a very wide range of bright sunshine (BSS) hours ranging from 1.0 hours to 9.6 hours. The highest bright sunshine hours (9.6) was recorded in 42-MW whereas the lowest sunshine hours recorded (1.0) in 27-MW. The highest wind velocity (6.2 kmph) was recorded in 32-MW and lowest wind velocity $(2.2 \mathrm{kmph})$ recorded in 42-MW respectively.

\section{Seed yield $\left(\mathrm{kg} \mathrm{ha}^{-1}\right)$}

The data presented in below table 1 indicated that mean grain yield was $163.88 \mathrm{Kg} \mathrm{ha}^{-1}$. 
Mean weekly weather data during experimental period 2016 at Parbhani

\begin{tabular}{|c|c|c|c|c|c|c|c|c|c|c|}
\hline \multicolumn{11}{|c|}{$\begin{array}{l}\text { WEEKLY WEATHER DATA RECORDED AT CENTRAL } \\
\text { METEOROLOGICAL OBSERVATORY PARBHANI } 2016\end{array}$} \\
\hline \multirow{2}{*}{ WK } & \multirow{2}{*}{ Period } & \multirow[t]{2}{*}{$\mathrm{RF}$} & \multirow[t]{2}{*}{$\mathrm{RD}$} & \multicolumn{2}{|c|}{ Temperature $\left({ }^{0} \mathrm{C}\right)$} & \multicolumn{2}{|c|}{ Humidity (\%) } & \multirow{2}{*}{$\begin{array}{l}\text { EVP } \\
(\mathrm{mm})\end{array}$} & \multirow{2}{*}{$\begin{array}{l}\text { BSS } \\
\text { (Hrs) }\end{array}$} & \multirow{2}{*}{$\begin{array}{c}\text { WV } \\
(\mathrm{kmph}\end{array}$} \\
\hline & & & & $\operatorname{Max}$ & Min & RH I & RH II & & & \\
\hline 27 & $2-8$ July & 80.2 & 5 & 30.1 & 23.9 & 85 & 73 & 3.8 & 1.0 & 5.9 \\
\hline 28 & $9-15 J u l y$ & 143.8 & 3 & 28.8 & 22.4 & 93 & 76 & 3.0 & 2.9 & 5.9 \\
\hline 29 & 16-22 July & 12.9 & 2 & 32.5 & 22.9 & 86 & 61 & 4.7 & 5.1 & 5.0 \\
\hline 30 & 23-29July & 65.6 & 3 & 30.7 & 22.9 & 93 & 68 & 3.7 & 4.1 & 4.4 \\
\hline 31 & 30 July-5 Aug. & 117.0 & 4 & 28.2 & 22.6 & 96 & 81 & 2.5 & 2.4 & 5.1 \\
\hline 32 & 6-12 Aug & 0.0 & 0 & 31.7 & 22.1 & 84 & 56 & 5.2 & 5.9 & 6.2 \\
\hline 33 & 13-19 Aug & 11.2 & 1 & 31.8 & 21.4 & 85 & 58 & 5.0 & 6.6 & 5.4 \\
\hline 34 & 20-26 Aug & 13.0 & 2 & 32.4 & 21.4 & 87 & 52 & 5.2 & 8.0 & 4.4 \\
\hline 35 & 27 Aug.-2 Sept. & 71.5 & 5 & 31.0 & 22.5 & 92 & 68 & 4.2 & 4.2 & 3.6 \\
\hline 36 & 3-9 Sept. & 1.5 & 0 & 30.9 & 20.7 & 80 & 58 & 5.3 & 8.8 & 5.0 \\
\hline 37 & 10-16 Sept. & 101.6 & 3 & 29.3 & 22.4 & 88 & 78 & 3.6 & 1.7 & 4.6 \\
\hline 38 & 17-23 Sept. & 109.1 & 4 & 29.6 & 22.3 & 96 & 85 & 2.9 & 2.5 & 3.4 \\
\hline 39 & 24-30 Sept. & 96.9 & 4 & 30.2 & 21.7 & 91 & 73 & 3.3 & 3.7 & 4.7 \\
\hline 40 & $1-7 \mathrm{Oct}$ & 109.5 & 3 & 29.4 & 21.3 & 93 & 72 & 3.4 & 5.3 & 3.9 \\
\hline 41 & 8-14 Oct & 56.9 & 2 & 32.0 & 21.2 & 88 & 52 & 4.7 & 7.5 & 2.9 \\
\hline 42 & $15-210 c t$ & 0.0 & 0 & 32.2 & 16.9 & 77 & 33 & 5.5 & 9.6 & 2.2 \\
\hline 43 & $22-28 \mathrm{Oct}$ & 0.0 & 0 & 32.3 & 16.1 & 74 & 31 & 5.9 & 9.0 & 2.7 \\
\hline 44 & 29 Oct.-4 Nov. & 0.0 & 0 & 31.2 & 14.4 & 75 & 32 & 5.1 & 9.5 & 3.8 \\
\hline & Total & 990.7 & 41 & & & - & & 77 & 97.8 & \\
\hline & Mean - & & & 31.79 & 21.06 & 86.8 & 61.5 & & - & 79.1 \\
\hline
\end{tabular}

Table.1 Mean seed yield of sesamum as influenced by different treatments

\begin{tabular}{|c|c|}
\hline \multirow[t]{2}{*}{ Treatments } & \\
\hline & Yield $\left(\mathrm{kgha}^{-1}\right)$ \\
\hline \multicolumn{2}{|l|}{ Sowing dates (D) } \\
\hline D1: $27^{\text {th }}$ MW & 173.71 \\
\hline D2: $28^{\text {th }}$ MW & 226.55 \\
\hline D3: $29^{\text {th }}$ MW & 130.33 \\
\hline D4: $30^{\text {th }}$ MW & 124.98 \\
\hline $\mathrm{SE} \pm$ & 5.34 \\
\hline CD at $5 \%$ & 18.49 \\
\hline \multicolumn{2}{|l|}{ Varieties (V) } \\
\hline V1 Phule til 1 & 152.75 \\
\hline V2 Gujarat 2 & 122.61 \\
\hline V3 JLT 408 & 203.23 \\
\hline V4 JLT 7 & 176.98 \\
\hline $\mathrm{SE} \pm$ & 13.04 \\
\hline CD at $5 \%$ & 45.14 \\
\hline \multicolumn{2}{|l|}{ Interaction $(\mathrm{D} x \mathrm{~V})$} \\
\hline $\mathrm{SE} \pm$ & 24.34 \\
\hline CD at $5 \%$ & NS \\
\hline General Mean & 163.88 \\
\hline
\end{tabular}


Table.2 Correlation between weather parameters and sesamum varieties seed yield at different phenophases

\begin{tabular}{|c|c|c|c|c|}
\hline \multirow[t]{2}{*}{ weather parameters } & \multicolumn{4}{|c|}{ Phenological stages of sesamum } \\
\hline & P1 & $\mathrm{P} 2$ & P3 & $\mathrm{P} 4$ \\
\hline \multicolumn{5}{|c|}{ Phule Til- 1} \\
\hline Rainfall & $0.500 *$ & $0.809 * *$ & $-0.795^{*}$ & 0.164 \\
\hline Rainy days & $0.826 * *$ & $0.984 * *$ & $-0.537 *$ & $0.952 * *$ \\
\hline Tmaxi & $-0.871 * *$ & 0.227 & $-0.683 * *$ & 0.098 \\
\hline Tmini & $-0.873 * *$ & -0.124 & $-0.978 * *$ & 0.205 \\
\hline RH -I & 0.420 & $0.862 * *$ & $0.560 *$ & $0.913 * *$ \\
\hline RH-II & $0.733 * *$ & 0.401 & 0.483 & $0.993 * *$ \\
\hline BSS & $-0.976^{* *}$ & 0.130 & $-0.990 * *$ & $-0.973 * *$ \\
\hline Wind speed & $-0.844 * *$ & $0.609 *$ & $-0.913^{*}$ & 0.205 \\
\hline \multicolumn{5}{|c|}{ Gujarat - 2} \\
\hline Rainfall & $0.683 * *$ & 0.216 & -0.492 & $0.513 *$ \\
\hline Rainy days & $0.847 * *$ & $0.988 * *$ & $0.607 *$ & $1.000 * *$ \\
\hline Tmaxi & $-0.842 * *$ & 0.015 & -0.490 & $-0.800 * *$ \\
\hline Tmini & $-0.900 * *$ & -0.119 & $-0.787 * *$ & $-0.570 *$ \\
\hline RH -I & 0.420 & $0.902 * *$ & $0.685^{* *}$ & $0.970 * *$ \\
\hline RH-II & $0.726 * *$ & 0.422 & $0.903 * *$ & $0.998 * *$ \\
\hline BSS & $-0.974 * *$ & $-0.894 * *$ & $-0.879 * *$ & $-0.995 * *$ \\
\hline Wind speed & $-0.886^{* *}$ & 0.143 & $-0.949 * *$ & -0.116 \\
\hline \multicolumn{5}{|c|}{ JLT- 408} \\
\hline Rainfall & $0.772 * *$ & $-0.639 * *$ & $-0.638 * *$ & $0.733 * *$ \\
\hline Rainy days & $0.931 * *$ & $0.974 * *$ & $0.834 * *$ & $0.994 * *$ \\
\hline Tmaxi & $-0.814 * *$ & $-0.529 *$ & $-0.587 *$ & $-0.985 * *$ \\
\hline Tmini & $-0.930 * *$ & -0.471 & $0.977 * *$ & $-0.934 * *$ \\
\hline RH -I & 0.387 & $0.840 * *$ & $-0.513^{*}$ & $0.989 * *$ \\
\hline RH-II & $0.544^{*}$ & $0.504 *$ & $0.630 * *$ & $0.999 * *$ \\
\hline BSS & $-0.995 * *$ & $-0.930 * *$ & $-0.975^{* *}$ & $-0.996 * *$ \\
\hline Wind speed & $-0.873 * *$ & $0.694 * *$ & -0.435 & $-0.901 * *$ \\
\hline \multicolumn{5}{|c|}{ JLT- 7} \\
\hline Rainfall & $0.628 * *$ & $-0.519^{*}$ & -0.035 & 0.414 \\
\hline Rainy days & $0.878 * *$ & $0.987 * *$ & 0.396 & $1.000 * *$ \\
\hline Tmaxi & $-0.724 * *$ & $-0.517 * *$ & -0.333 & $-0.924 * *$ \\
\hline Tmini & $-0.870 * *$ & -0.460 & $-0.915 * *$ & $-0.741 * *$ \\
\hline RH -I & 0.054 & $0.844 * *$ & $0.915^{* *}$ & $0.978 * *$ \\
\hline RH-II & 0.333 & 0.451 & $0.980^{* *}$ & $0.999 * *$ \\
\hline BSS & $-0.997 * *$ & $-0.935^{* *}$ & $-0.909 * *$ & $-0.983 * *$ \\
\hline Wind speed & $-0.919 * *$ & $0.588^{*}$ & $-0.806 * *$ & $-0.565^{*}$ \\
\hline
\end{tabular}

(* Significant at $5 \%, * *$ Significant at $1 \%$ )

Phenological stages of Sesamum

$\mathrm{P}_{1}$ : Sowing to Vegetative Stage

$\mathrm{P}_{2}$ : Vegetative Stage to Reproductive Stage

$\mathrm{P}_{3}$ : Reproductive Stage to Maturity Stage

$\mathrm{P}_{4}$ : Maturity Stage to Harvesting Stage 


\section{Effect of sowing dates}

The crop sown at $28^{\text {th }}$ MW produced significantly highest seed yield $(226.55 \mathrm{~kg}$ $\mathrm{ha}^{-1}$ ) than rest of the sowing dates, whereas significantly the lowest seed yield was observed under $30^{\text {th }}$ MW. This might be the effect of optimum temperature, good rainfall and BSS (hrs) during crop growth period under former sowing date (Ali et al., 2005). The lowest seed yield was observed at $30^{\text {th }}$ MW because of the terminal drought during reproductive phase. Similar results were reported by Choudhary et al., (2015).

\section{Effect of varieties}

The variety JLT - 408 recorded significantly higher seed yield $\left(203.23 \mathrm{~kg} \mathrm{ha}^{-1}\right)$ than JLT-7 $\left(176.98 \mathrm{~kg} \mathrm{ha}^{-1}\right)$, Phule til-1 (152.75 kg ha $\left.{ }^{-1}\right)$ and Gujarat $-2\left(122.61 \mathrm{~kg} \mathrm{ha}^{-1}\right)$. This might be due to less flower drop, more number of branches and more number of filled capsules plant $^{-1}$ with former variety. The minimum grain yield $122.61 \mathrm{~kg} \mathrm{ha}^{-1}$ was observed in variety Gujarat -2 . The results are confirmed by Jadhav et al., (2015) with their investigation.

\section{Effect of interaction}

The effect of interaction between sowing dates and varieties at harvest were found nonsignificant for grain yield.

\section{Correlation studies of yield}

The rainfall, rainy days, RH -I, RH-II, wind speed has been positively correlated with sesamum variety Phule Til-1 seed yield at $\mathrm{P} 1$, $\mathrm{P} 2, \mathrm{P} 2$ to $\mathrm{P} 4, \mathrm{P} 1$ and $\mathrm{P} 4, \mathrm{P} 2$ stage respectively; otherwise at P1 Tmax. Tmin, BSS, wind speed; at P3 all weather parameters excepted $\mathrm{RH}-\mathrm{I}, \mathrm{RH}-\mathrm{II}$ and at $\mathrm{P} 4$ stage only BSS has been negatively correlated (Table 2).
In case of variety Gujarat-2, the weather parameters was positively correlated with seed yield was rainfall at $\mathrm{P} 1$ and $\mathrm{P} 2$; rainy days at all stages; RH -I at P2, P4; RH-II at P2, P3, P4 stage where as negatively correlated was Tmax at P1 and P4; Tmin at P1, P3, P4; BSS at all stages and wind speed at $\mathrm{P} 1$ and $\mathrm{P} 3$ stage.

At P1 stage rainfall, rainy days, $\mathrm{RH}-\mathrm{II}$; at $\mathrm{P} 2$ rainy days, $\mathrm{RH}-\mathrm{I}, \mathrm{RH}-\mathrm{II}$, wind speed; at $\mathrm{P} 3$ rainy days, Tmin, RH-II and at $\mathrm{P} 4$ stage rainfall, rainy days, $\mathrm{RH}-\mathrm{I}$, RH-II was positively correlated with the seed yield of sesamum variety JLT- 408 and negatively correlated weather parameters was Tmax., Tmin, BSS, wind speed at P1; Rainfall, Tmax. BSS at P2; Rainfall, Tmax. RH-I, BSS, wind speed at P3 and Tmax. Tmin, BSS, wind speed at $\mathrm{P} 4$ stage.

The rainfall has positively correlated at P1 and negatively correlated at P2 stage; Rainy days positively correlated at $\mathrm{P} 1, \mathrm{P} 2, \mathrm{P} 4$; and Tmax. Tmin was negatively correlated at all stage; RH -I, RH-II was positively correlated at all stages; however BSS and wind speed has negatively correlated at all stage i.e. P1, P2, P3, P4 with seed yield of sesamum variety JLT-7.

\section{References}

Anonymous (2015). Area, Production and Productivity.www.mahaagri.org.in

Asghar Ali, Asif Tanveer, Muhammad Athar Nadeem, and Amir Liaquat Bajwa (2005). Effect of sowing dates and row spacings on growth and yield of sesame. J. Agric. Res.43(1).

Choudhary, C.S., Arun, Anjana and Prasad, S.M. (2015). Influence of dates on sowing and weather parameters incidence and development of Alternaria leaf spot of sesame, Internat. J. Pl. Protec., 8(1): (73-76). 
Jadhav, M.G., Chaudhary, G.B., Patil, T.R., and Patil, S.C. (2015). JLT-408 A new high yielding sesame variety for Maharashtra state. Electronic J. Plant
Breeding, 6(1):118-124.

Snedecor, C.W., and W.G. Cochran (1968).

Statistical methods. Oxford and I.B.H.

Publication Co. Bombay. Pp. 585

\section{How to cite this article:}

Sawant, D.M., U.N. Alse, B.V. Asewar and Khobragade, A.M. 2019. Correlation Studies between Weather Parameters and Sesamum (Sesamum indicm L.) Varieties Yield at Different Phenophases under Change in Climate. Int.J.Curr.Microbiol.App.Sci. 8(05): 997-1002. doi: https://doi.org/10.20546/ijcmas.2019.805.117 